\title{
Properties of Vacuum Arcs Generated by Switching RMF Contacts at Different Ignition Positions
}

\author{
Sergey Gortschakow ${ }^{1, *} \mathbb{D}^{\mathbb{D}}$, Steffen Franke $\left.{ }^{1} \mathbb{(}\right)$, Ralf Methling ${ }^{1}{ }^{(0}$, Diego Gonzalez ${ }^{1}$, \\ Andreas Lawall ${ }^{2}$, Erik D. Taylor ${ }^{2}$ and Frank Graskowski ${ }^{2}$ \\ 1 Department of Plasma Radiation Techniques, Leibniz Institute for Plasma Science and Technology, \\ 17489 Greifswald, Germany; steffen.franke@inp-greifswald.de (S.F.); methling@inp-greifswald.de (R.M.); \\ diego.gonzalez@inp-greifswald.de (D.G.) \\ 2 Siemens AG, 13629 Berlin, Germany; andreas.lawall@siemens.com (A.L.); \\ erik-d.taylor@siemens.com (E.D.T.); frank.graskowski@siemens.com (F.G.) \\ * Correspondence: sergey.gortschakow@inp-greifswald.de
}

Received: 17 September 2020; Accepted: 21 October 2020; Published: 26 October 2020

\begin{abstract}
The influence of initiation behavior of the drawn arc on the arc motion, on arc characteristics during the active phase, as well as on the post-arc parameters, was studied. The study was focused on arc dynamics, determination of the anode surface temperature after current interruption, and diagnostics of metal vapor density after current zero crossing. Different optical diagnostics, namely high-speed camera video enhanced by narrow-band optical filters, near infrared spectroscopy, and optical absorption spectroscopy was applied. The initiation behavior of the drawn arc had a clear influence on arc parameters. Higher local electrode temperature occurs in case of the electrodes with ignition point near the outer electrode boundary. This further causes an enhanced density of chromium vapor, even in cases with lower arc duration. The results of this study are important for design development of switching RMF contacts for future green energy applications.
\end{abstract}

Keywords: vacuum interrupter; switching arc; optical diagnostics

\section{Introduction}

Vacuum technology provides environmentally compatible and emission-free solutions for switching applications in power grids [1,2]. Some advantages of vacuum circuit breakers include a high number of operations under standard load conditions, safe and reproducible short-circuit current interruption capability, and maintenance-free operation. Further development of such devices requires fundamental knowledge about the switching process and interaction of materials used with the working medium - the vacuum arc plasma.

The vacuum arc is ignited by the electrode separation under the current load. While the arc appears as diffuse glow for currents typically below $10 \mathrm{kA}$ [1-3], constriction effects dominate at higher currents. The disadvantage of a constricted arc is that it causes a localized thermal load on the electrode, which leads to enhanced electrode erosion due to melting and evaporation. Therefore, various measures are applied in switching devices for arc control. The application of external magnetic fields helps either to hold the arc in diffuse stage (axial magnetic field (AMF)) or causes the rotation of the constricted arc (radial magnetic field (RMF)) or transverse magnetic field (TMF)) [1].

The RMF/TMF contacts have been in use for many decades. In the pioneering works [4-6], the two different contact shapes were suggested. Corresponding principles are used in the modern contact systems in vacuum interrupters and give inspiration for improved contact geometries [7]. The choice of contact design depends on the electrical and thermal load in a certain application. 
In case of successful current interruption, the arc is terminated immediately after the current zero (CZ) crossing. However, there are various factors that could prevent the current interruption. When the density of neutral metal vapor is high enough, ionization due to appearance of transient recovery voltage can lead to arc reignition and disconnection failure [8]. The anode surface is the main source of neutral vapor. The anode activity is particularly high if high-current anode modes occur [9]. In case of RMF contacts, the arc movement prevents formation of stationary anode spots. However, the arc needs certain time to maintain the magnetic field for the start of arc rotation. During this stage, the arc position is fixed, which leads to a localized electrode heating. In later stages, the same position could be repeatedly heated, due to a high rotation speed. Since thermal dissipation is a relatively slow process, the position of arc initiation can potentially reach higher temperatures compared to the surrounding material and thereby influence the performance of the contacts. Thus, studying the influence of arc ignition position on the arc dynamics, spatio-temporal evolutions of electrode surface temperature, and vapor density after current interruption is of great practical importance for the understanding of switching behavior and development/improvement of the contact design.

Optical diagnostics offer numerous methods for the characterization of the arc plasma and electrodes. High-speed camera techniques are widely used for observation of arc dynamics, such as appearance of certain high-current modes, mode transitions, arc constriction, rotation speed, etc., [10-13]. Various high-speed cameras are used for acquisition of temporal evolution of light emitted by the arc (mainly in visible range). Typically, an acquisition frequency of $5 \mathrm{k}-100 \mathrm{k}$ fps is applied.

Optical emission spectroscopy (OES) can determine the surface temperature. A big challenge for surface temperature measurements is the presence of the arc in front of the electrode. The arc radiation is quite strong during the whole arcing phase and disappears immediately after current zero crossing. This behavior of plasma radiation gives the opportunity for undisturbed access to the electrode surface after the current zero. The corresponding measurements techniques have been reported in [14-16]. One of the possibilities is to use a compact near infrared spectrometer [14], which is adjusted to the position of interest by an optical system. The method works as long as the surface emits enough thermal radiation.

Evaluation of the ground state density of neutral species ( $\mathrm{Cu}$ and $\mathrm{Cr}$ atoms) can be performed by optical absorption spectroscopy (OAS) [17-21]. The absorption measurement setup typically consists of a light source, optical system for beam adjustment and a spectrograph for registration of absorption spectra. Usually, broadband light sources are applied since they provide enough power in the visible spectral region and, thus, allow for a choice of various wavelength ranges of interest. The measurements are of feasible complexity as long as they are performed after the current zero crossing, when the emission from arc plasma is negligibly small [17-20]. Investigations in the active phase, however, require much more effort, e.g., arrangement of a second optical path for registration of plasma radiation from the probed region only [21]. This technique is very complicated and works well in the case of non-moving arc only. Therefore, a setup with single optical path was used for measurements after current interruption.

In the present study, the influence of the arc ignition position on local surface temperature of the anode and vapor density after current zero crossing was investigated in a model vacuum interrupter using the above-mentioned optical diagnostics under realistic operation conditions.

\section{Materials and Methods}

\subsection{Experimental Setup}

Figure 1 presents the experimental setup, comprising a model vacuum interrupter (vacuum chamber 1 with pneumatic drive 2), a high-current generator (visible in the background of Figure 1), as well as electrical and optical diagnostics (3-8).

A vacuum chamber with optical access described in [22] has been used. It contains four optical viewports allowing for simultaneous acquisition of several optical signals. The electrodes are moved 
by a pneumatic drive with an opening speed of about $1 \mathrm{~m} / \mathrm{s}$. The fixed electrode was powered as an anode. The maximum stroke of the drive system was about $10 \mathrm{~mm}$. The electrode distance at which the arc interruption occurs depends on the opening speed and the instant of contact separation start and is typically $3-8 \mathrm{~mm}$.

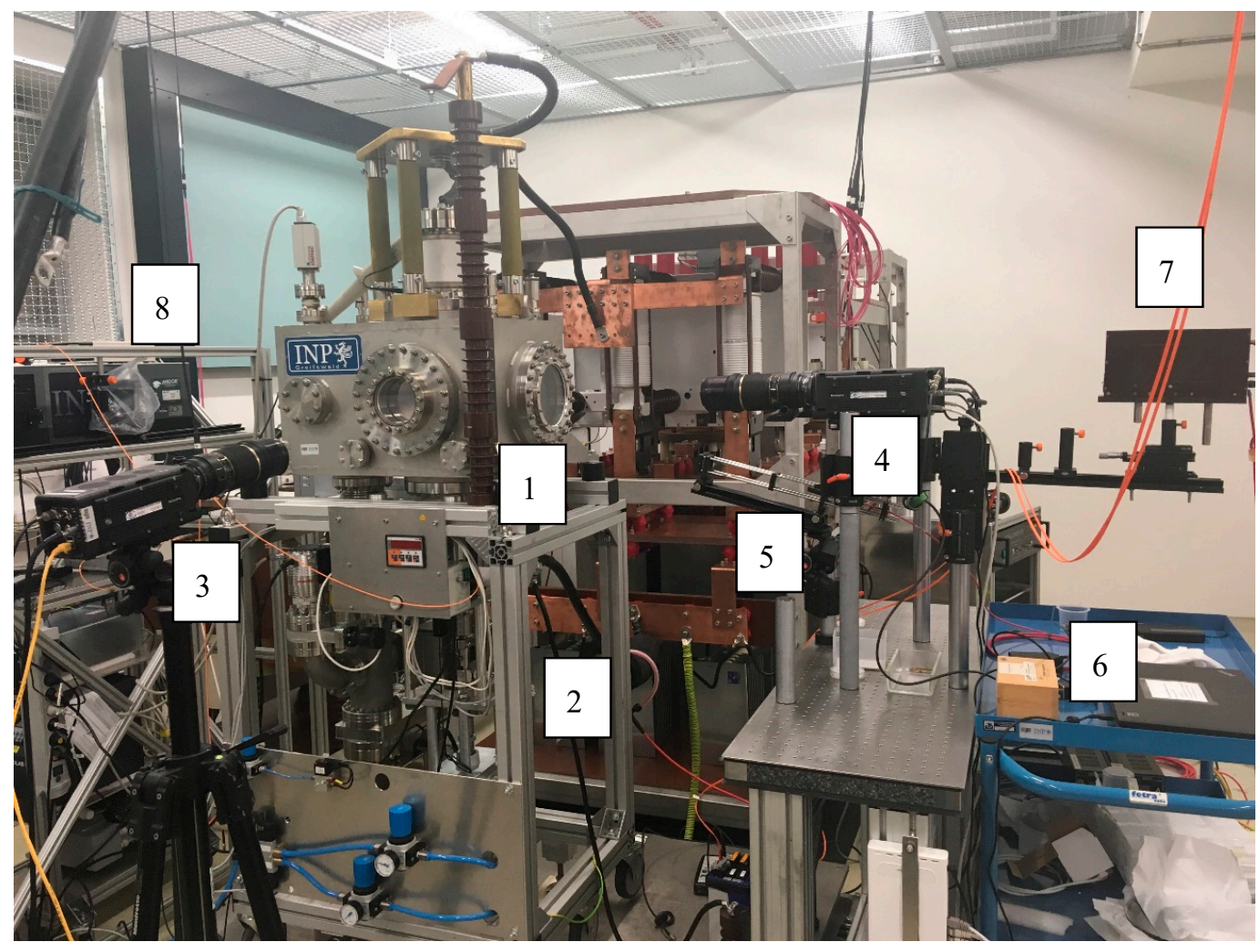

Figure 1. Setup for studies on vacuum arcs: (1) vacuum chamber, (2) pumping system, (3) high-speed camera (general arc dynamics), (4) two high-speed cameras equipped with narrow-band optical filters, (5) NIR optics, (6) NIR spectrometer, (7) Xe flash lamp, (8) 0.75 m imaging spectrograph.

\subsection{Power Source}

Two different power sources have been used. For electrode surface cleaning a pulsed DC current of $400 \mathrm{~A}$ and $20 \mathrm{~ms}$ duration has been applied [22]. The second power generator, providing $50 \mathrm{~Hz}$ equivalent current with a magnitude of about $28 \mathrm{kA}$ (peak value), was used for the main experiments. The arc duration varied between 3 and $8 \mathrm{~ms}$, depending on the time instant of electrode separation.

\subsection{Electrodes}

Spiral RMF electrodes made of $\mathrm{CuCr}$ alloy were used. Electrode diameter was $34 \mathrm{~mm}$. Contact spots at defined positions for arc initialization have been created mechanically. Figure 2 shows examples of prepared electrodes.

These initial contact spots had diameters of about $3 \mathrm{~mm}$. Only the cathodes were modified in order to reduce the influence on the anode as much as possible

Figure 3 demonstrates the working principle of a RMF contact pair. When the electrode separation starts, the last connection point between the electrodes serves as an arc ignition position due to fast local overheating (bridge explosion). The arc column constricts due to the action of self-induced magnetic field. The spiral form of the contact produces an additional magnetic field, which pushes the arc toward the outer electrode boundary. As far as the constricted arc column reaches the outer boundary, the joint action of the current flow (blue tracer in Figure 3) and magnetic field (green arrows in Figure 3) causes a Lorentz force (direction is shown by red arrows in Figure 3), which rotates the arc. 


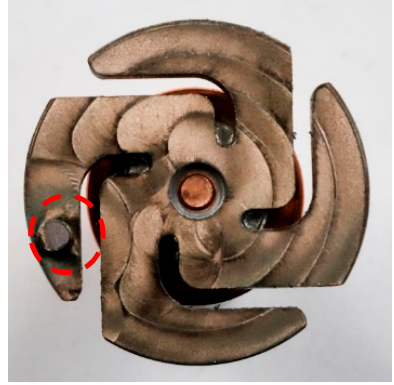

(a)

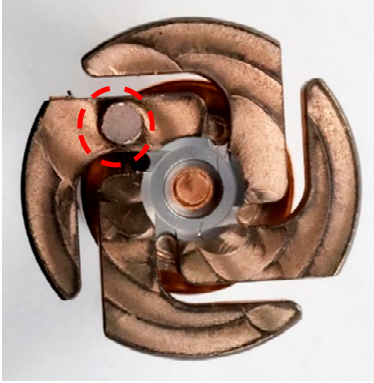

(b)

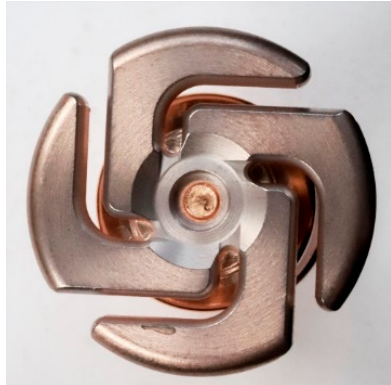

(c)

Figure 2. Examples of $\mathrm{CuCr}$ electrodes used in the study. Red circles show the prepared contact spots for arc ignition, type A (a) and type B (b). The anode (c) was not modified.

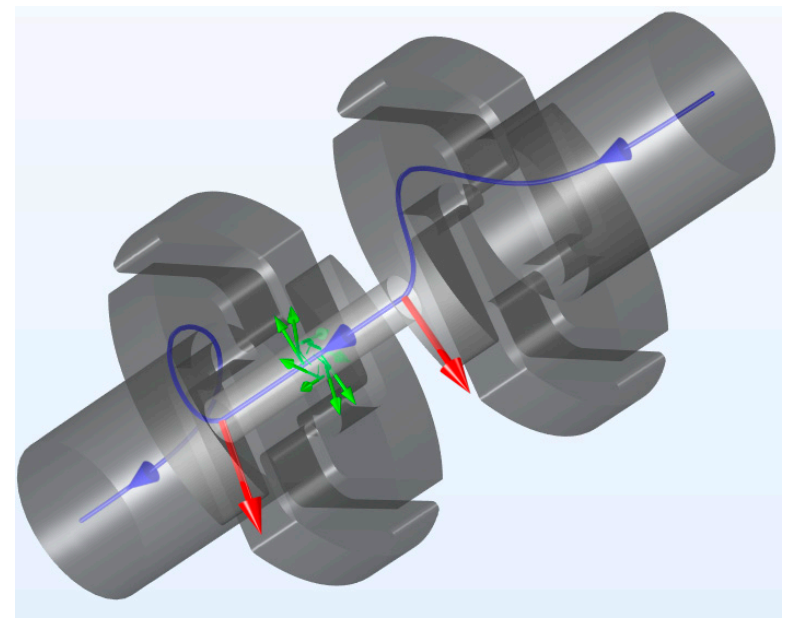

Figure 3. Schematic representation of RMF contact working principle. Blue tracer shows the current flow, green arrows represent the direction of magnetic flux density, and red arrows show the direction of resulting Lorentz force.

\subsection{Diagnostics}

Electrical and optical diagnostics was used for characterization of the arc behavior. Several high-speed cameras acquired the arc dynamics (Figure 1). Near infrared (NIR) spectroscopy determined the time-resolved anode surface temperature after current interruption. Broadband absorption spectroscopy in turn determined the vapor density with respect to different initial arc positions.

\subsubsection{Electrical Diagnostics}

Arc current and arc voltage have been measured by corresponding probes. A Rogowski current probe (model CWT 1500, PEM) measured the current evolution. The arc voltage was acquired using a high voltage probe (P6015A, Tektronix). The signals were registered by a transient recorder (GEN7t, HBM).

\subsubsection{Arc Images}

Several high-speed cameras registered the arc dynamics (Motion Pro Y4, IDT) with a repetition/frame rate of 20,000 fps and an exposure time of $1 \mu \mathrm{s}$. Standard camera lenses were used. One camera monitored the ignition position and general arc dynamics. In addition, two cameras equipped with narrow-band metal-interference filters transmitting at $521 \mathrm{~nm}$ with FMHW $1 \mathrm{~nm}$ (Cu I line) and at $494 \mathrm{~nm}$ with FWHM $4 \mathrm{~nm}$ (several Cu II lines) separated the dynamics of copper atoms and ions during the arcing. 


\subsubsection{Determination of Surface Temperature}

Near infrared spectroscopic measurements (NIR in Figure 1) have been used for evaluation of the local anode surface temperature after current interruption (after the time instant $10 \mathrm{~ms}$ in Figure $4 \mathrm{~b}$ ) at the points of arc ignition. A NIR spectrometer (C1142GA, Hamamatsu) with a spectral range of $900-1650 \mathrm{~nm}$ and a temporal resolution of $1.25 \mathrm{~ms}$ (exposure time $200 \mu \mathrm{s}$ ) was used for spectral measurements. After the acquisition, the spectra were processed according to the routine presented in [14] to determine the anode surface temperature. Figure 4 summarizes the information about NIR diagnostics. The red spot in Figure 4a shows the region, from which the anode surface radiation was collected. The measurement spot was about $1 \mathrm{~mm}$ in diameter and focused on the arc ignition position, i.e., in case of electrodes of type A, the position shown by red circle in Figure 2a was used, in case of the electrodes of type B, the corresponding position shown in Figure $2 \mathrm{~b}$. The measurements started around the current maximum with an acquisition time of $200 \mu$ s and frequency of one spectrum each $1.25 \mathrm{~ms}$.

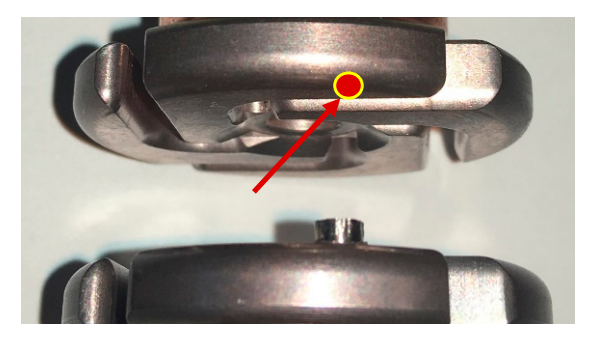

(a)

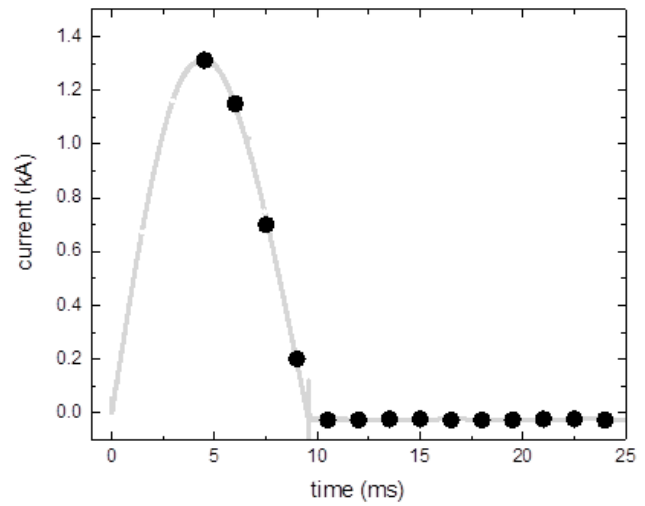

(b)

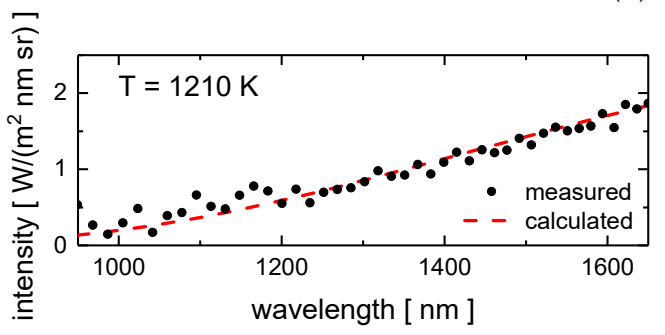

(c)

Figure 4. (a) Measurement position in case of NIR spectroscopy (red circle). Anode on the top, cathode in the bottom. (b) Acquisition instants of NIR spectroscopy related to the current shape. (c) Sample of NIR spectrum suitable for temperature evaluation: instant of time $1.25 \mathrm{~ms}$ after current interruption.

Figure $4 \mathrm{c}$ shows an example of temperature evaluation for $1.25 \mathrm{~ms}$ after current interruption (point around $11.25 \mathrm{~ms}$ in Figure $4 \mathrm{~b}$ ) for the contact pair of type B. The surface temperature was calculated using diagnostic techniques described in [14]. A tungsten strip lamp was used for determination of wavelength dependent windows transmission, as well as for spectral calibration of NIR spectrometer. The temperature arises from the shape of measured intensity (Figure 4c) by comparison with a Planck radiator with given temperature. Details of the methods are described in [14].

\subsubsection{Absorption Spectroscopy for Species Density Determination}

Broadband optical absorption spectroscopy (OAS) determined the chromium vapor density after extinction of arc plasma (after current interruption). This technique is based on evaluation of absorption spectra in the wavelength range where the resonance lines of material of interest are present.

A pulsed DC high-intensity Xenon lamp acted as a background radiation source. It emits a Planck-like radiation of 12,000 K with a maximum power of $1 \mathrm{MW}$ [23]. The Xenon lamp is positioned 
on the right-side window of the vacuum chamber (Figure 1). Its radiation is directed through the electrode system and coupled to the spectrometer entrance slit that is placed at the opposite window. By using a deflecting and a focusing mirror, the electrode gap is observed along a line parallel to the electrode surfaces (dashed line in Figure 5a). The slit width was about $50 \mu \mathrm{m}$. The spatial position of spectra acquisition was about $1 \mathrm{~mm}$ away from the anode surface. The lamp and the spectrograph start at a desired instant (Figure 5b). The Xe lamp started at about $9.5 \mathrm{~ms}$ (green curve in Figure 5b) and reached its maximum intensity close to the instant of acquisition time (grey curve denoted as OAS in Figure 5c). The radiation is spectrally dispersed using a Czerny-Turner type spectrograph (Shamrock 750, Andor Technology Ltd.) with a $0.75 \mathrm{~m}$ focal length equipped with an intensified charge coupled device camera (iStar, Andor Technology Ltd.). Absorption spectra were acquired immediately after the current zero crossing with a delay between $100 \mu \mathrm{s}$ and $300 \mu \mathrm{s}$, which was necessary to study the decaying plasma. A spectral interval between $423-431 \mathrm{~nm}$ was chosen to study the $\mathrm{Cr}$ I density, which contains spectral lines of different resonance transitions of $425.43,427.78$, and $428.97 \mathrm{~nm}$. Figure 4c presents an example of an acquired spectrum for Cr I $428.97 \mathrm{~nm}$ line. The density of absorbing species is proportional to the area under the curve. More details about the method and the corresponding theory are described in $[20,21]$.

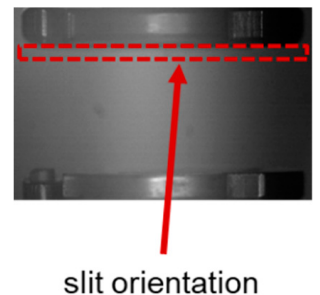

(a)

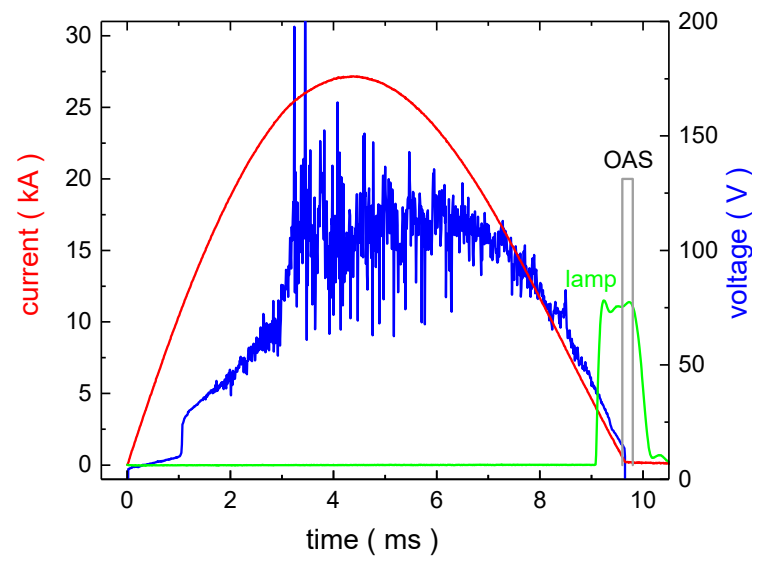

(b)

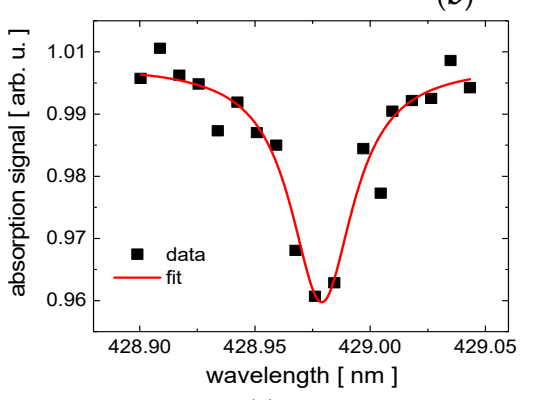

(c)

Figure 5. (a) Position of spectrograph slit for absorption spectra acquisition. Slit width was $50 \mu \mathrm{m}$; distance to the anode was about $1 \mathrm{~mm}$. (b) Temporal evolution of current (red) and voltage (blue) along with sequence of control signals during the absorption spectroscopy measurements: driving current of Xe lamp (green) and exposure signal for spectrum acquisition (grey). (c) Example of Cr I $428.97 \mathrm{~nm}$ line fit procedure.

\section{Results and Discussion}

\subsection{Electrode Preparation}

The switching contacts in real vacuum interrupters are manufactured under special conditions in a clean atmosphere [1]. These conditions are typically not available in research facilities. Therefore, some cleaning procedure must be applied prior to a study of arc properties in order to remove adsorbed 
micro particles, lubricant from the machining processing, dielectric layers, water, etc. The cleaning procedure comprises processing in an ultrasonic bath with distilled water and a degreasing fluid, surface cleaning with isopropanol, and application of low-current arc discharges $(<400 \mathrm{~A})$ of less than $30 \mathrm{~ms}$ duration for final conditioning of the surface after the mounting inside the vacuum chamber and pumping to ultra-high vacuum. First shots with non-conditioned electrodes show instabilities in the voltage behavior (Figure 6), which is related to stochastic spot formation on the electrode surface at the positions of adsorbed impurities. The spot formation is accompanied by voltage jumps (blue curve in Figure 6) in agreement with different arc voltages for cathode spots I and II [24]. The high-speed videos confirm the unstable arc root positions in this case. After typically 10 shots, the voltage curve becomes much smoother (green curve in Figure 6), indicating the end of the conditioning process.

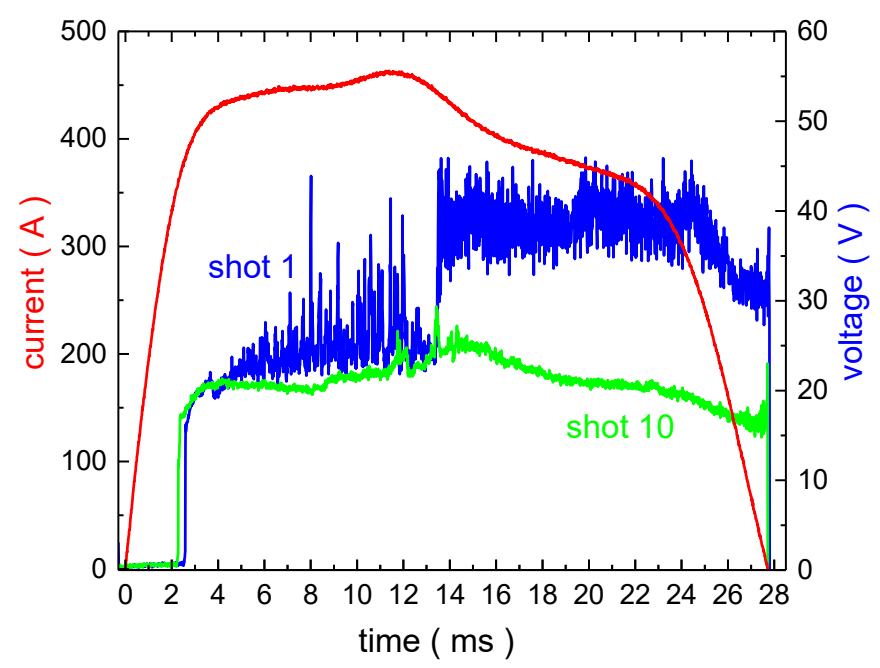

Figure 6. Electrical signals during the conditioning process. Red curve-arc current; blue and green curves-arc voltage during two different shots; blue curve before conditioning and green curve after conditioning.

\subsection{Arc Dynamics}

Figure 7 presents the typical evolutions of arc voltage and current along the discharge with spectrally filtered images. The ignition instant in the considered example (the start of electrode separation) was about $2.2 \mathrm{~ms}$ (Figure $7 \mathrm{~b}, \mathrm{c}$ ). The ignition position in present study was defined by mechanical surface modification, as described in Section 2.3. After the ignition, the arc spreads fast over an area, which is larger than the ignition spot and remains diffuse in the present example up to about $3.6 \mathrm{~ms}$ (Figure 7d,e). During the diffuse stage, the arc is formed by a nearly homogeneous distributed cathode spots, uniformly glowing anode and a diffuse arc column. The spatial distribution of atoms and ions is very similar at this stage. The bridge explosion gives enough material, which fills a significant volume due to small electrode distance. The arc becomes constricted around the last contact spot (ignition point) (Figure 7f,g) after a certain amount of time, which is necessary to build up enough electromagnetic force (Lorentz force) for constriction process. Notice that even after constriction, the cathode spots cover nearly the whole electrode surface. Their distribution becomes inhomogeneous with higher spot density around the position of constricted arc attachment. Opposite to this, the anode attachment is localized and moves in accordance with actual electromagnetic force direction. Thus, the arc rotation means moving group of cathode spots, opposite anode spot and the arc column, which connects both attachments. The arc trajectory (moving position of highest intensity) is close to the outer electrode boundary. The frames (h-k) show the instants of time when the arc was passing through the ignition position. The spatial distributions of atoms and ions start to differ from each other. At this stage, the atomic radiation is more constricted and has higher intensity near to the cathode surface, while the ionic radiation is distributed wider and more homogeneously 
over the gap region. This qualitative picture changes as soon as the arc starts to rotate, i.e., after about $3.6 \mathrm{~ms}$ in the present example. During rotation, the arc column is dominated by the ions. Their spatial distribution is homogeneous within the emitting area. The atoms follow the ions with some delay. The radiation remains most intense near the cathode surface and is less pronounced in the arc column. The area of atomic radiation near the cathode is much larger compared to that of ionic radiation. The cathode surface is nearly completely melted as will be shown below. Notice, that an arc constriction does not imply that the electrode surface outside of the constriction area is not active anymore. Detailed analysis of high-speed images shows that some weak cathode spots continue to exist on the broader electrode area, and thus represent an intense source of atoms. In addition, the atoms, which are evaporated by melted surface, can become excited due to reaction kinetic processes, like e.g., ion recombination, de-excitation cascades, or charge transfer reactions between copper species. Detailed explanation requires a space- and time-dependent collisional-radiative model, which is missing due to its complexity.

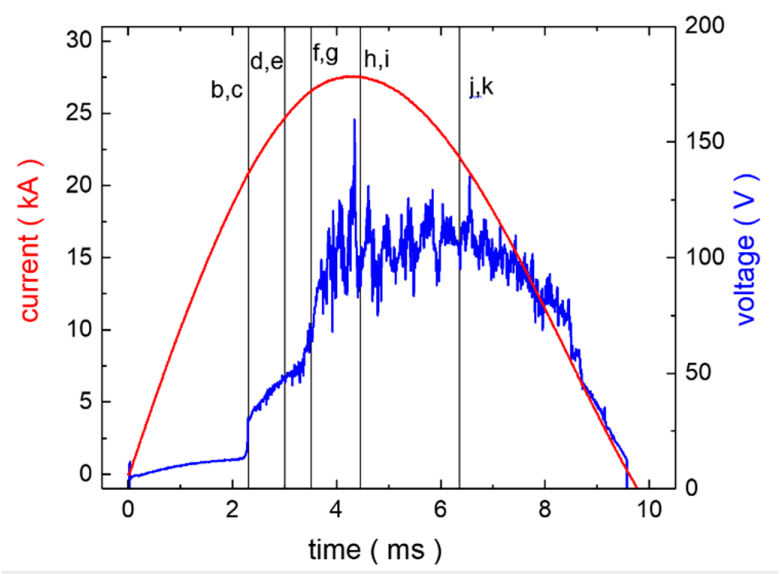

(a)

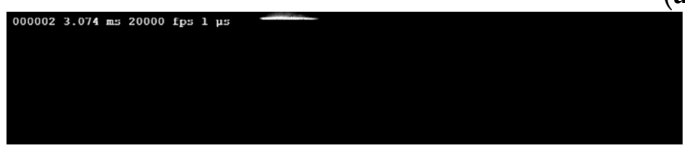

(b)

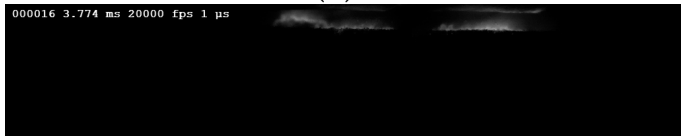

(d)

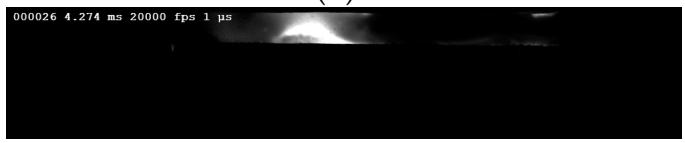

(f)

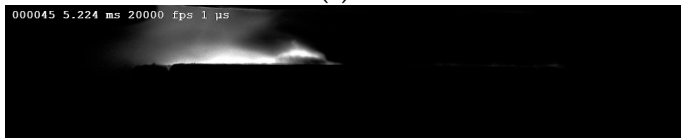

(h)

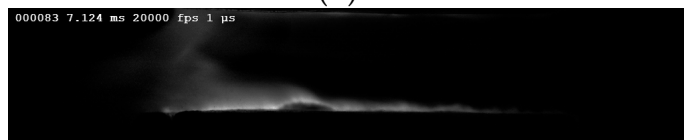

(j)

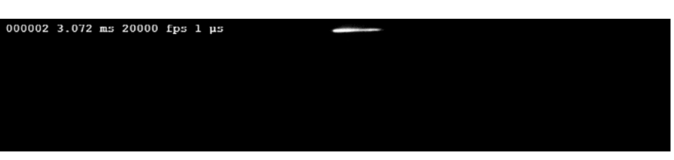

(c)

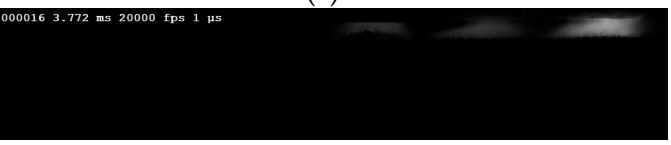

(e)

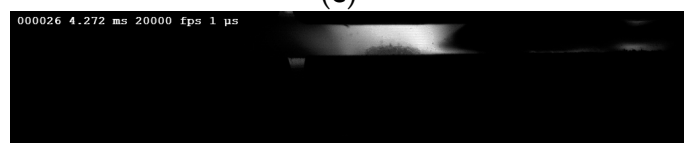

(g)

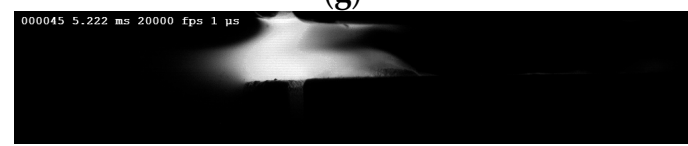

(i)

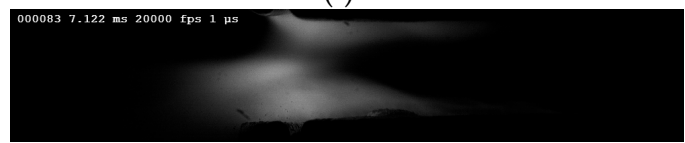

(k)

Figure 7. (a) Temporal evolution of arc current and voltage. Vertical lines show the instants for which the arc images are presented. (b-k) Spectrally filtered arc images at different time instants. Images $(\mathbf{b}, \mathbf{d}, \mathbf{f}, \mathbf{h}, \mathbf{j})$ show $\mathrm{Cu}$ I emission, $(\mathbf{c}, \mathbf{e}, \mathbf{g}, \mathbf{i}, \mathbf{k})$ that of $\mathrm{Cu}$ II. Electrode type A. 
Figure 8 presents the arc rotation frequency along with the arc current shape. It was determined from the arc images by evaluation of the instants at which the arc column passed the same spatial position on the electrode surface. The rotation frequency depends on several factors, like. e.g., arc current value, magnitude of magnetic field flux and the electrode distance. At the beginning, the rotation is slow. The frequency reaches its maximum value, which is about $4 \mathrm{kHz}$ in current example, around the peak current. Then, the frequency follows the current, decreasing up to the instant of about $6.5 \mathrm{~ms}$. The rotation becomes slightly faster for the later time instants, due to accumulated effects of phase shift between the arc current and induced magnetic flux and increasing electrode distance. The initial arc position has no influence on rotation frequency taking into account the accuracy of applied method. In the case of position B (which is closer to the electrode centre), the arc starts to move closer to the electrode axis and reaches the outer rotational position slightly later, comparing to ignition position A. The analysis of the high-speed images clarifies that most of the electrode surface becomes molten (or at least close to the molten state) after several rotation cycles. Thus, for the same arc duration a comparable molten surface area will be reached later for the case with ignition position B.

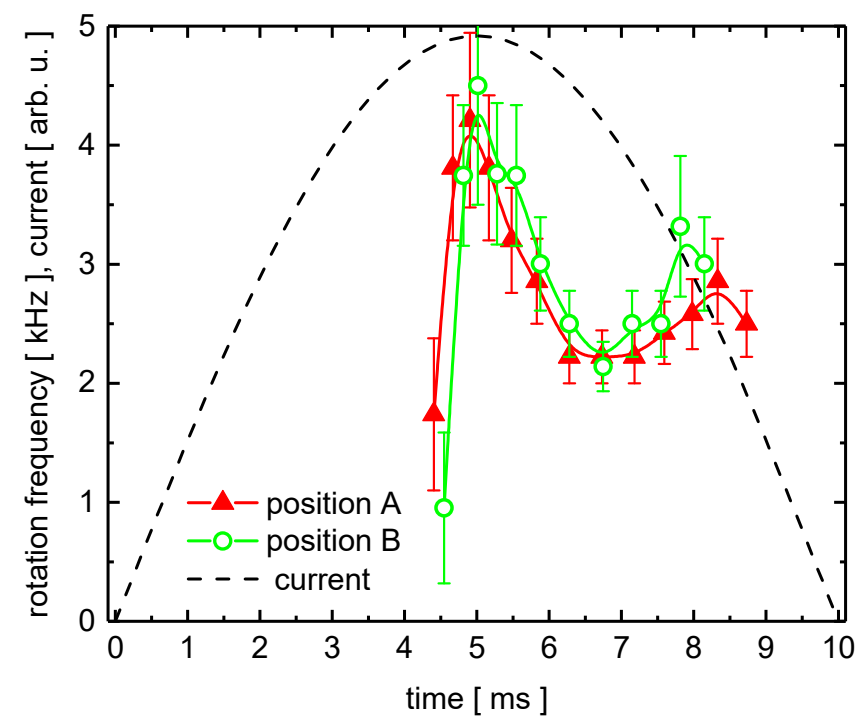

Figure 8. Rotation frequency of the arc determined from high-speed images. Red triangles-contact system of type A; green circles-those of type B. Arc current evolution is presented by dashed curve.

\subsection{Anode Surface Temperature}

Figure 9 presents the temporal evolutions of local anode surface temperature after the current zero crossing at the position of arc ignition, i.e., at localized spot of about $1 \mathrm{~mm}$ in diameter. In case of electrodes of type A, the position shown in Figure 2a was used, in case of the electrodes of type B, the position shown in Figure $2 \mathrm{~b}$. Notice that the temperature in the other regions of the anode surface can significantly differ from obtained values. However, it is expected that measured values give an estimate for the maximum anode temperature after current interruption, since the accumulated arc residence time in those region is higher than in surrounding regions. For type A electrodes, the initial temperature tends to increase with the arc duration. Its initial value was around $1300 \mathrm{~K}$. Longer arcing time leads to slower temperature decay. Since the arc is rotating, the measurement point could be slightly cooled down between two subsequent rotations. However, with increasing arc duration the amount of melted material becomes bigger. This is clearly visible in the videos. Consequently, the melted pool needs more time for cooling down. The type B electrodes show less variability in the temperature. The position at which the temperature was measured is in this case closer to the electrode axis. The arc residence time in that region is much shorter comparing to the outer region. Consequently, the temperature is lower, its value is about $1200 \mathrm{~K}$. Slower temperature decay in this region is mainly caused by contact design. The cooling path for the surface is going toward the stem. 
The electrode temperature is obviously higher in the outer regions (Figure 9). Thus, those regions act as a heat source for the inner part of the electrode after current interruption. Therefore, a temperature stagnation occurs in those regions.

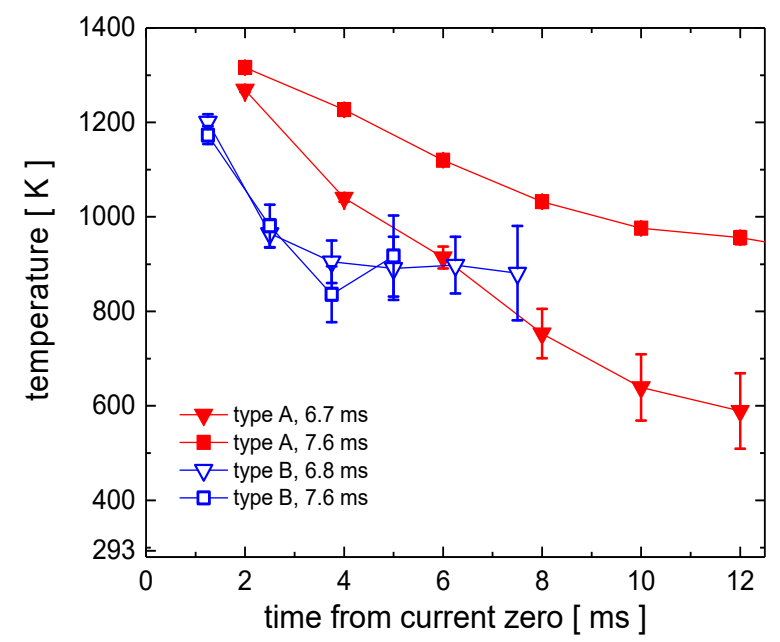

Figure 9. Evolution of surface temperature measured at ignition position after the arc extinction. Arc duration time is indicated. Uncertainty of the numerical method is shown. Total measurements uncertainty is estimated as $\pm 50 \mathrm{~K}$ [14].

\subsection{Cr Density after Current Interruption}

Figure 10 shows the results of absorption measurements for $\mathrm{Cr}$ I vapor density after current zero crossing. Due to low spectrally resolved radiation intensity, the acquisition of only one spectrum per shot is possible. Therefore, several measurements (up to five for each contact pair) at the same peak current value of $28 \mathrm{kA}$ have been performed. Due to the presence of many mechanical parts in the driving and in the switching systems it is not possible to get exactly the same arc duration in different shots and the same instant of absorption spectrum acquisition. On the other hand, the reproducibility of plasma characteristics seems to be quite stable, when the same arc duration occurs. Thus, different shots give an estimation of temporal evolution after current zero crossing. The temporal region within first $500 \mu \mathrm{s}$ is of special importance for interruption process. During this time period, the fast increasing transient recovery voltage can force a breakdown if the species densities are high enough. Atomic species do not follow the electric field and have enough residence time between the electrodes to get ionized. The density of neutral species has the highest value direct after the current zero crossing instant and shows a nearly linear decay within the first microseconds after current interruption [20,21].

The arc duration has an insignificant influence on $\mathrm{Cr}$ density only. Initial temperatures at current zero do not differ much for the same contact type (cf. Figure 9). The density slightly increases with longer arc duration. Stronger deviations in the density were found when the arc ignition position was varied. A higher electrode temperature leads consequently to higher density of chromium vapor in the case of the electrodes with position A. It amounts to about $1.1 \times 10^{18} \mathrm{~m}^{-3}$ for starting position $\mathrm{A}$ immediately after current interruption, while the value for position B is about $9.5 \times 10^{17} \mathrm{~m}^{-3}$, even though the arc duration was longer. The results are similar for other time instants. 


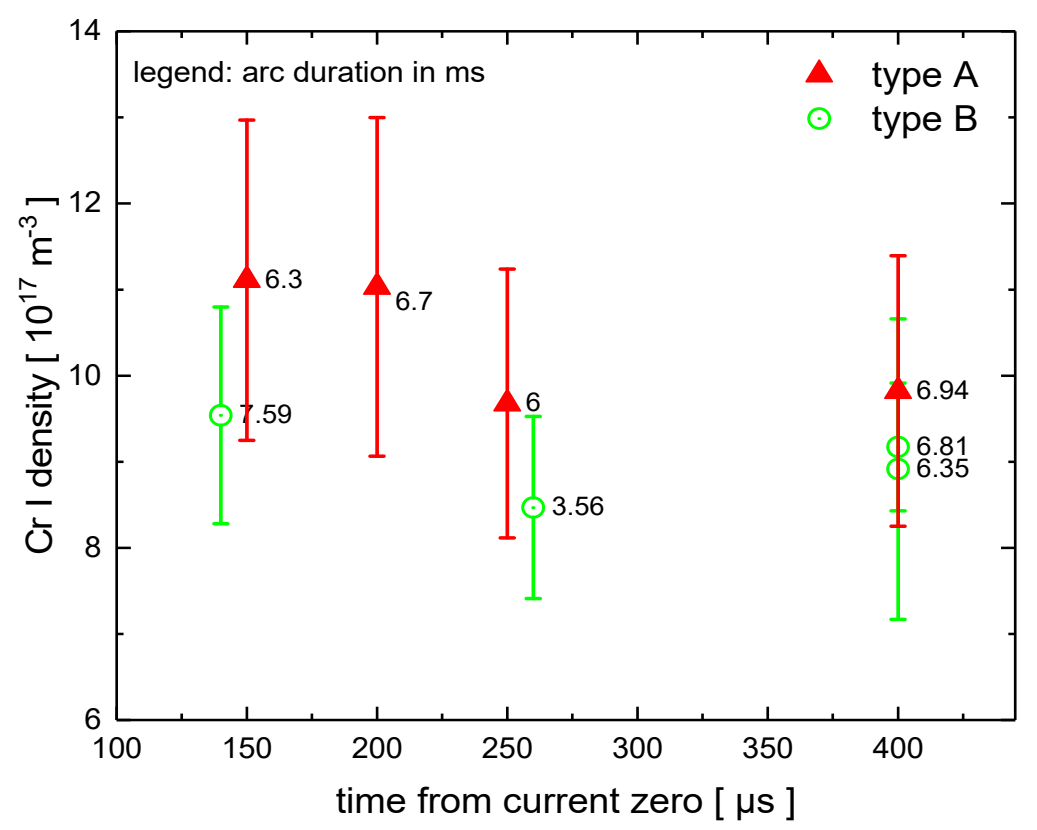

Figure 10. Cr I density measured after current zero crossing for different shots with contact systems of type A (red symbols) and B (green symbols). The numbers indicate the arc duration in ms.

\section{Summary}

The conducted study clarified that there is a clear influence of initiation behavior of the drawn arc on the electrode temperature, which is about $100 \mathrm{~K}(8 \%)$ higher in case of outer radial position measured directly after current interruption. Cr vapor density after current interruption is about $10 \%$ higher for outer radial positions. Therefore, it can be concluded that arc ignition in outer regions leads to higher local anode temperature and mean vapor density. Such behavior could potentially lead to increased electrode erosion, shorter lifetimes, and more restrikes (breakdown after current interruption). Future electrode design should take into account possible changes in electrode surface topology after significant current load.

Further investigations are planned to study the dependence of obtained results on material (electrode composition), as well other electrode design (such as AMF contacts).

Author Contributions: Conceptualization, S.G., A.L., E.D.T., F.G.; methodology, S.G., R.M., S.F.; software, S.F., S.G.; validation, E.D.T., F.G.; formal analysis, S.G.; investigation, S.G., D.G.; resources, D.G.; data curation, S.G.; writing-original draft preparation, S.G.; writing—review and editing, S.F., R.M., D.G., A.L., E.D.T. and F.G.; visualization, S.G., R.M. and S.F.; supervision, D.G. and F.G.; project administration, S.G. and A.L. All authors have read and agreed to the published version of the manuscript.

Funding: This research received no external funding.

Conflicts of Interest: The authors declare no conflict of interest.

\section{References}

1. Slade, P.G. The Vacuum Interrupter: Theory, Design, and Application; CRC Press: Boca Raton, FL, USA, 2008.

2. Giere, S.; Hellig, D.; Koletzko, M.; Kosse, S.; Rettenmaier, T.; Stiehler, C.; Wenzel, N. Vacuum interrupter unit for $\mathrm{CO}_{2}$-neutral 170kV/50kA switchgear. In ETG-Fb. 157: VDE-Hochspannungstechnik 2018; 12.-14.11.2018; VDE Verlag GmbH: Berlin, Germany, 2018; pp. 28-31.

3. Lippmann, H.J. Schalten im Vakuum, Physik und Technik der Vakuumschalter; VDE Verlag GmbH: Berlin, Germany, 2003.

4. Schneider, H.N. Contact Structure for an Electric Circuit Interrupter. U.S. Patent 2,949,520, 19 August 1958.

5. Lake, A.A.; Reece, M.P. Improvements Relating to Vacuum Switch Contact Assemblies. UK Patent 997,384, 7 July 1965. 
6. Hundstad, R.L. Contact Structures for Vacuum-Type Circuit Interrupters Having Cantilevered-Supported Annularly-Shaped Outer Arc-Running Contact Surfaces. U.S. Patent 3,845,262, 29 October 1974.

7. Lamara, T.; Hencken, K.; Gentsch, D. A Novel Vacuum Interrupter Contact Design for Improved High Current Interruption Performance Based on a Double-TMF Arc Control System. IEEE Trans. Plasma Sci. 2015, 43, 1798-1805. [CrossRef]

8. Dullni, E.; Schade, E. Investigation of high-current interruption of vacuum circuit breakers. IEEE Trans. Electr. Insul. 1993, 28, 607-620. [CrossRef]

9. Miller, H.C. Anode modes in vacuum arcs: Update. IEEE Trans. Plasma Sci. 2017, 45, 2366-2374. [CrossRef]

10. Schulman, M.B.; Schellekens, H. Visualization and characterization of high-current diffuse vacuum arcs on axial magnetic field contacts. IEEE Trans. Plasma Sci. 2000, 28, 443-451. [CrossRef]

11. Shang, W.; Dullni, E.; Fink, H.; Kleberg, I.; Schade, E.; Shmelev, D.L. Optical investigations of dynamic vacuum arc mode changes with different axial magnetic field contacts. IEEE Trans. Plasma Sci. 2003, 31, 923-928. [CrossRef]

12. Gentsch, D.; Shang, W. High-speed observations of arc modes and material erosion on RMF- and AMF-contact electrodes. IEEE Trans. Plasma Sci. 2005, 33, 1605-1610. [CrossRef]

13. Wolf, C.; Kurrat, M.; Lindmayer, M.; Wilkening, E.-D.; Gentsch, D. Optical investigations of high-current vacuum arc behavior on spiral-shaped and cup-shaped RMF-contacts. In Proceedings of the 55th IEEE Holm Conference on Electrical Contacts, Vancouver, BC, Canada, 14-16 September 2009; pp. 270-275.

14. Methling, R.; Franke, S.; Gortschakow, S.; Abplanalb, M.; Sütterlin, R.-P.; Delachaux, T.; Menzel, K.O. Anode surface temperature determination in high current vacuum arcs by different methods. IEEE Trans. Plasma Sci. 2017, 45, 2099-2107. [CrossRef]

15. Chaly, A.M.; Poluyanova, I.N.; Yakovlev, V.V.; Zabello, K.K.; Logatchev, A.A.; Shkol'nik, S.M. Experimental study of anode surface temperature after current zero for a range of current levels. In Proceedings of the 27th International Symposium on Discharges and Electrical Insulation in Vacuum (ISDEIV), Suzhou, China, 18-23 September 2016; pp. 1-4. [CrossRef]

16. Logachev, A.A.; Poluyanova, I.N.; Zabello, K.K.; Begal, D.I.; Shkol'nik, S.M. Analysis of cathode surface state and cathode temperature distribution after current zero of AMF-contacts. In Proceedings of the 28th International Symposium on Discharges and Electrical Insulation in Vacuum (ISDEIV), Greifswald, Germany, 23-28 September 2018; pp. 329-332.

17. Gortschakow, S.; Popov, S.; Khakpour, A.; Schneider, A.; Methling, R.; Franke, S.; Uhrlandt, D. Cu and Cr density determination during high-current discharge modes in vacuum arcs. In Proceedings of the 28th International Symposium on Discharges and Electrical Insulation in Vacuum(ISDEIV), Greifswald, Germany, 23-28 September 2018; pp. 181-184.

18. Horvath, B.; Lamara, T. Time-resolved optical resonant absorption spectroscopy of $\mathrm{Cr}$ metallic vapor in air using a broadband LED light source. Plasma Sources Sci. Technol. 2013, 22, 035006. [CrossRef]

19. Wang, H.; Wang, Z.; Liu, U.J.; Zhou, Z.; Wang, J.; Geng, Y.; Liu, Z. Optical absorption spectroscopy of metallic (Cr) vapor in a vacuum arc. J. Phys. D Appl. Phys. 2018, 51, 035203. [CrossRef]

20. Khakpour, A.; Popov, S.; Franke, S.; Kozakov, R.; Methling, R.; Uhrlandt, D.; Gortschakow, S. Determination of $\mathrm{Cr}$ density after current zero in a high-current vacuum arc considering anode plume. IEEE Trans. Plasma Sci. 2017, 45, 2108-2114. [CrossRef]

21. Gortschakow, S.; Khakpour, A.; Popov, S.; Franke, S.; Methling, R.; Uhrlandt, D. Determination of Cr density in a high-current vacuum arc considering anode activity. Plasma Phys. Technol. 2017, 4, 190-193. [CrossRef]

22. Methling, R.; Gorchakov, S.; Lisnyak, M.V.; Franke, S.; Khakpour, A.; Popov, S.; Batrakov, A.; Uhrlandt, D.; Weltmann, K.-D. Spectroscopic investigation of high-current vacuum arcs. In Proceedings of the 26th 2014 International Symposium on Discharges and Electrical Insulation in Vacuum (ISDEIV), Mumbai, India, 28 September-3 October 2014; pp. 221-224. 
23. Günther, K.; Radtke, R. A proposed radiation standard for the visible and UV region. J. Phys. E 1975, 8, 371-376. [CrossRef]

24. Boxman, R.L.; Martin, P.J.; Sanders, D.M. Handbook of Vacuum Arc Science and Technology; Noyes: Hammonton, NJ, USA, 1995.

Publisher's Note: MDPI stays neutral with regard to jurisdictional claims in published maps and institutional affiliations.

(C) 2020 by the authors. Licensee MDPI, Basel, Switzerland. This article is an open access article distributed under the terms and conditions of the Creative Commons Attribution (CC BY) license (http://creativecommons.org/licenses/by/4.0/). 